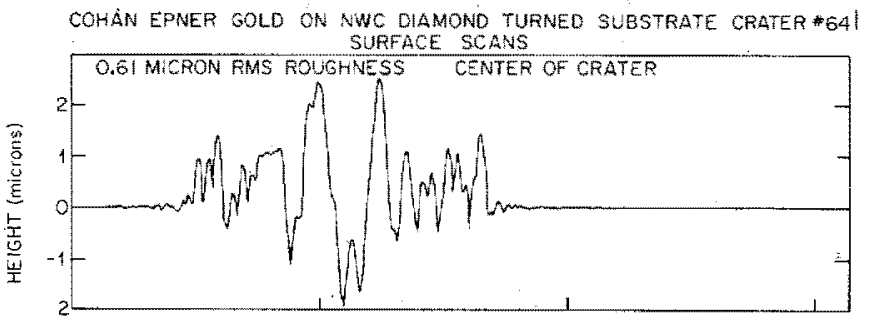

(a)

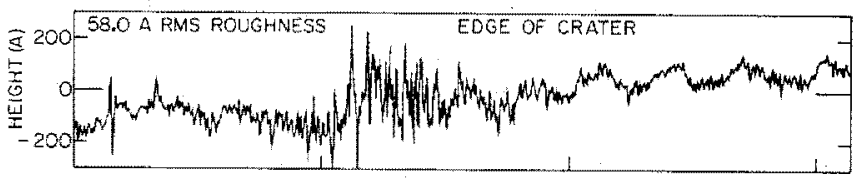

(b)

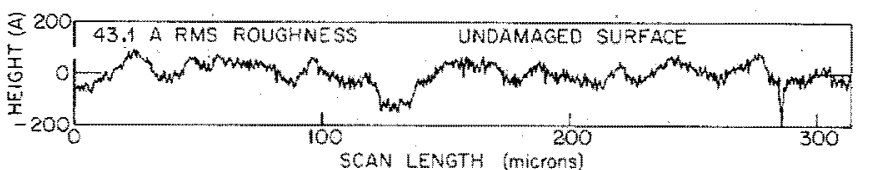

(c)

Fig. 4. Surface profle of Aurelectroplated diamond-turned Cu. Part (a) shows the surface profile of a catastrophically damaged area, i,e., the crater, part (b) shows the surface profile of the roughened area, both irradiated with 0.532 um light. Part (c) shows the surface profile of the unirradiated area.

irregularities may enhance the local optical electric field strength and thereby reduce the damage resistance of the site.

\section{ACKNOWLEDGMENT}

The authors would like to thank Dr. J. M. Bennett, Michaelson Laboratory, Naval Weapons Center for making the surface roughness measurements and Dr. L. M. Merkle for helpful discussions: In addition, they would like to thank Dr. D. M.
Decker (NWC), the Intop Corporation, and the Northrop Corporation for providing the samples.

\section{REFERENCES}

[1] Y. R. Shen, "Recent advances in non-linear optics," Rev. Mod. Phys, vol. 48, pp. 1-32, Jan, 1976, and references therein.

[2] S. Roberts, "Optical properties of copper," Phys. Rev., vol. 118, pp. 1509-1518, June 1960.

[3] P. B. Johnson and R. W. Christy, "Optical constants of copper and nickel as a function of temperature," Phys. Rev. B, vol. 11, pp. 1315-1323, Feb. 1975.

[4] J. A. Mackay and J. A. Rayne, "Temperature dependence of the infrared absorptivity of the noble metals," Phys. Rev. B, vol. 13, pp. 673-684, Jan. 1976.

[5] D. L. Decker and V. A. Hodgkin, Wavelength and Temperature Dependence of the Absolute Reflectance of Metals at Visible and Infrared Wavelengths. Nat. Bur. Stand. Spec. Publ, vol. 620, pp. $190-193,1980$.

[6] N. Koumvakalis, C. S. Lee, and M. Bass, "Intensity dependent absorption and laser induced catastrophic damage in diamond turned and mechanically polished Cu mirrors at $1.06 \mathrm{\mu m}$," in Proc. 13th Symp. on Laser Induced Damage in Optical Materials, Boulder, CO, Nov, 1981, and to be published by the National Bureau of Standards.

[7] C. S. Lee, N. Koumvakalis, and M. Bass, "Spot-size dependence of laser-induced damage to diamond turned Cu mirrors," Appl. Phys. Lett., vol. 41, pp. 625-627.

[8] M. Sparks and E. Loh, Jr., "Temperature dependence of absorp. tance in laser damage of metallic mirrors: L. Melting," $J$. Opt. Soc. Amer, vol. 69, pp. 847-858, June 1974.

[9] J. M. Bennet and J. H. Daney, "Stylus profiling instrument for measuring statistical properties of smooth optical surfaces," Appl. Opt., vol. 20, pp. 1785-1802, May 1981.

[10] Handbook of Chemistry and Physics, 59 th ed. CRC, 1978.

[11] R. S. Quimby, M. Bass, and L. Liou, "Calorimetric measurement of temperature dependent absorption in copper," in Proc. 13th Symp. on Laser Induced Damage in Optical Materials, Boulder, CO, Nov. 1981, and to be published by the National Bureau of Standards.

[12] N. Bloembergen, "Role of cracks, pores and absorbing inclusions on laser induced damage threshold at surfaces of transparent dielectrics,"Appl. Opt., vol. 12, pp. 661-664, Apr. 1973.

\title{
Compact Hg II Laser Excited by a Transverse Electron Beam Glow Discharge
}

\author{
JORGE J. ROCCA, DAVID M. MC CLURE, AND GEORGE J. COLLINS
}

\begin{abstract}
CW laser action at an output power of $6 \mathrm{~mW}$ has been obtained on the 6149.5 \& transition of $\mathrm{Hg} \mathrm{II}$, using a $10 \mathrm{~cm}$ long transverse electron bean glow discharge. Beam energies are typically $1 \mathrm{keV}$. Laser tube design, discharge characteristics, and laser output power are reported.
\end{abstract}

W E have obtained $\mathrm{CW}$ laser action on the $6149.5 \AA$ transition of $\mathrm{Hg}$ II ising a $10 \mathrm{~cm}$ long transverse electron beam excited glow discharge. We previously reported

Manuscript received March 21, 1983; revised May 13, 1983. This work was supported by the National Science Foundation and the AFOSR.

J. J. Rocea and G. J. Collins are with the Department of Electrical Engineering, Colorado State University, Fort Collins, CO 80523.

D. M. McClure was with the Department of Electrical Engineering, Colorado State University, Fort Collins, $\mathrm{CO}$. He is now with the Department of Applied Physics, Columbia University, New York, NY, the generation of $\mathrm{CW}$ laser radiation in several singly ionized species in He-metal vapor mix tures using longitudinal election beam excitation [1]-[5]. In this excitation scheme, a glow discharge electron gun [6] produces an electron beam with an energy between 1 and $5 \mathrm{keV}$ that is guided by an axial magnetic field to generate a laser active medium $50-100 \mathrm{~cm}$ long. In the transverse excitation scheme we describe below, the magnetic field is not needed to ensure efficient deposition of the electron beam energy into the gas mixture. Rather, electrostatic fields confine and partially trap the beam electrons. This allows for the compact laser geometry shown schematically in Figs. 1 and 2(a).

A new cathode material is used in our glow discharge electron gun. This is a sintered mixture of molybdenum and magnesium oxide particles (10 $\mu \mathrm{m}$ diameter) in equal proportions 


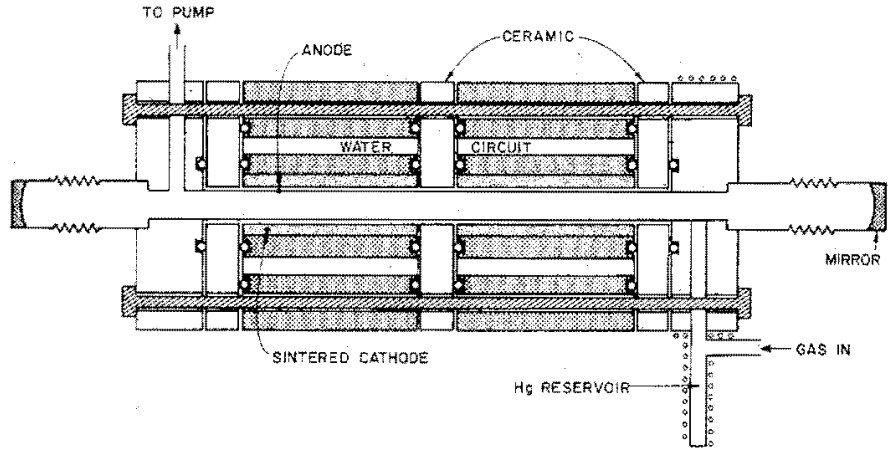

Fig, 1. Schematic diagram of the transverse electron beam laser. For clarity, details such as complete water-cooling circuit are now shown.

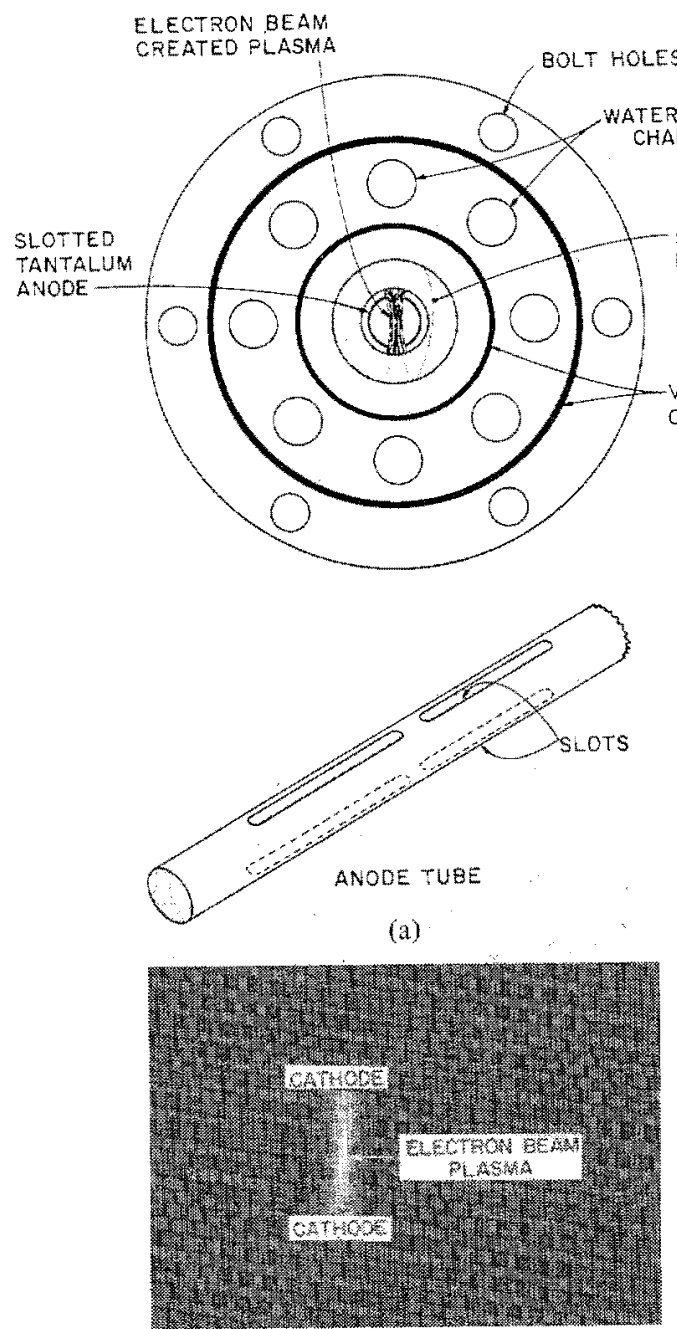

(b)

Fig. 2. (a) Schematic end view of a cathode segment and slotted anode, (b) End view of the electron beam glow discharge.

by weight. The magnesium oxide particles have a high secondary electron emission coefficient $\gamma$ following ion bombardment, making the cathode an efficient electron emitter [7]. The molybdenum particles are necessary to make the material an electrical conductor suitable for de plasma operation. In contrast to the cathodes coated with either an aluminum or magnesium oxide film, as described in [6], the sintered cathodes we employ in this study can work in a pure noble gas or even a metal vapor-noble gas mixture without requiring the addition of oxygen to maintain cathode emissivity.

Hollow cylindrical pieces of the sintered material, $11 \mathrm{~mm}$ inside diameter and $7 \mathrm{~cm}$ long, were press fit into water-cooled copper pieces. Two of these cathode segments were separated by a single piece of machinable ceramic, as shown in Fig. 1. Each cathode was connected to the de power supply through a separate ballast resistor. The two stainless steel end pieces are also separated from the cathode segments by machinable ceramic spacers. O-rings were used as a vacuum seal between pieces. The stainless steel end pieces support a slotted tantalum tube which acts as the concentric anode. The anode surface is approximately $0.5 \mathrm{~mm}$ from the cathode and has two sets of opposing slots $0.5 \mathrm{~cm}$ wide and $5 \mathrm{~cm}$ long, as shown in Fig. 2(a), through which the electron beam passes.

The anode tube also covers the regions between different pieces and consequently protects the O-ring seals from the electron beam created discharge. The $0.5 \mathrm{~mm}$ distance between the electrodes, under operating conditions (pressures between 1 and 6 torr) is less than the mean free path for an ionizing electron collision. For an electron with an energy near the peak value of the total cross section $(120 \mathrm{eV})$ for ionization of helium $\left(\sigma=3.7 \times 10^{-17} \mathrm{~cm}^{2}\right)$, the corresponding mean free path is $5 \mathrm{~mm}$ at a helium pressure of 6 torr and a gas temperature of $1000^{\circ} \mathrm{C}$. Higher electron energies and lower pressures have even larger mean free paths for ionization. Consequently, there is not sufficient ionization in the annular interelectrode region to establish a stable discharge. In this way, a constricted glow discharge with a length of $10 \mathrm{~cm}$ is created. The electron beam is produced by acceleration in the cathode dark space of the electrons emitted following cathode bombardment by ions and fast neutrals. Practically all the discharge voltage $V_{c}$ drops in this cathode fall region, creating an electron beam of energy approximately equal to $e \times V_{c}$. The concave cathode geometry focuses the electron beam towards the axis of the discharge volume. The beam electrons lose part of their energy in exciting and ionizing collision as they travel towards the opposite cathode surface. The beam electrons are then reflected by the electric field in the opposite dark space [8]. The opposing concave cathode surfaces allow for both the trapping [8] and focusing of the beam electrons, and consequently efficient deposition of beam energy into the gas mixture occurs. The electron beam, electrostatically trapped in this way, creates a bright plasma with the clear boundries as shown in Fig. 2(b). Transverse high voltage glow discharges have been obtained in the past [9], [10] by similar techniques but with lower $\gamma$ cathode materials, and without allowing for electrostatic trapping of the beam electrons.

Mercury vapor was introduced into the discharge tube by heating a reservoir located in one of the stainless steel end pieces. Helium was introduced at the same end of the discharge tube and was flowed at a rate of 8000 SCCM via a roughing pump. The optical cavity was formed using two internally mounted dielectric mirrors of $2 \mathrm{~m}$ radius of curvature.

The $I-V$ characteristics of the transverse electron beam glow discharge in helium are shown in Fig. 3. Stable discharges at 


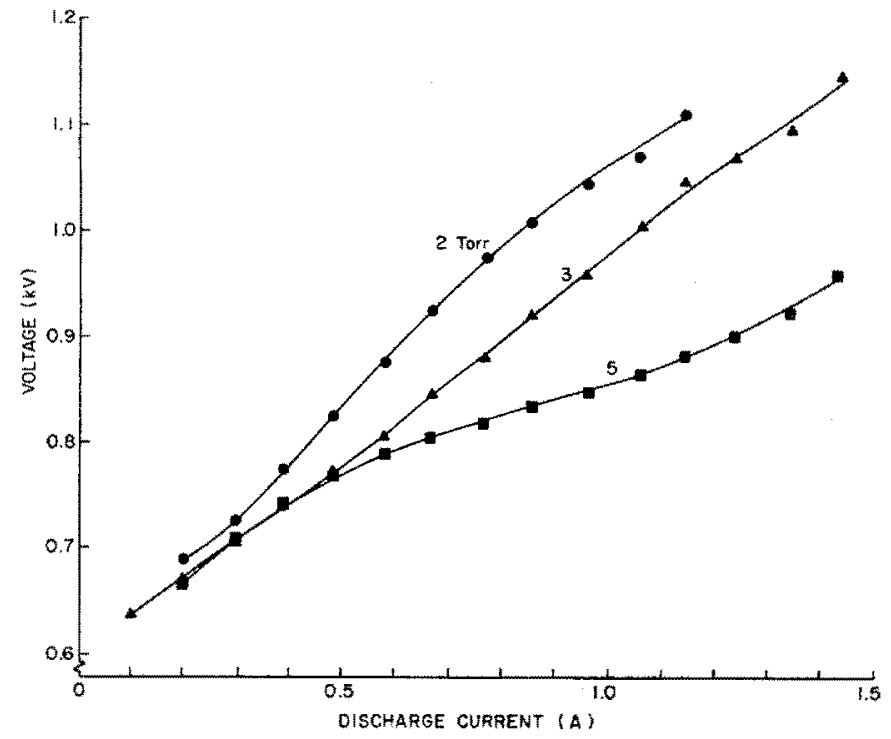

Fig. 3, I-V characteristics of the transverse electron beam glow discharge in helium using two cathode segments.

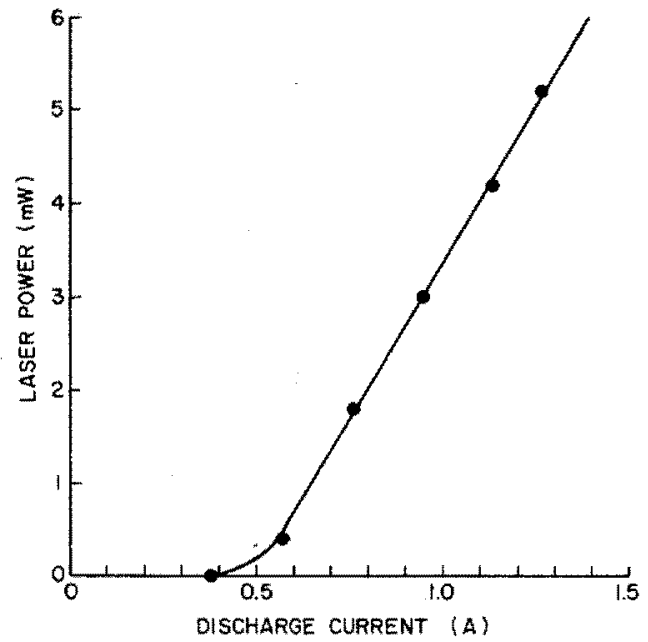

(a)

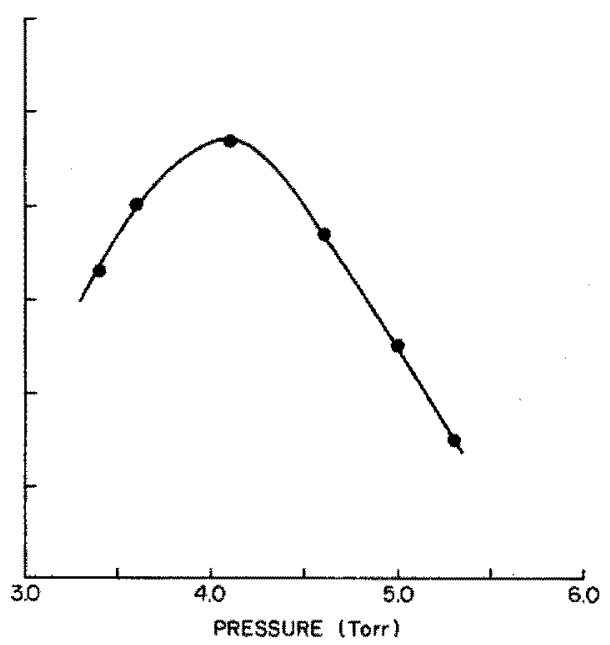

(b)

Fig. 4. (a) Laser output power as a function of discharge current. Helium pressure was 4 torr. $\mathrm{Hg}$ reservoir temperature was $180^{\circ} \mathrm{C}$. (b) Laser output power as a function of helium pressure for a discharge current of $1.3 \mathrm{~A}$. Temperature of the $\mathrm{Hg}$ reservoir was $180^{\circ} \mathrm{C}$,

currents over $1 \mathrm{~A}$ and at a voltage of $1 \mathrm{kV}$ have been obtained using two cathode segments, each having an effective discharge length of $5 \mathrm{~cm}$. The laser output power versus discharge current is shown in Fig. 4(a) for a helium pressure of 4 torr. The laser output power increases linearly with current and no saturation was observed up to the maximum discharge current investigated. A CW laser output power of $6 \mathrm{~mW}$ was obtained using an unoptimized output coupler of 99.8 percent reflectivity at $6150 \AA$. Fig. 4(b) shows the variation of the laser output power as a function of helium pressure for a discharge current of $1.3 \mathrm{~A}$. The dominant excitation mechanism is proposed to be charge transfer excitation of the $\mathrm{Hg}$ II $7 \mathrm{p}^{2} \mathrm{P}^{\circ}{ }_{3 / 2}$ level from the helium ion ground state He II $1 \mathrm{~s}^{2} \mathrm{~S}_{1 / 2}$ [11]. The $10 \mathrm{~cm}$ long discharge, of modular construction, can be extended adding more cathode segments to obtain larger laser powers.

In summary, we have obtained $\mathrm{CW}$ laser action in the 6149.5
$\hat{\AA}$ transition of $\mathrm{Hg}$ II using a $10 \mathrm{~cm}$ long transverse electron beam glow discharge to excite a $\mathrm{He}-\mathrm{Hg}$ mixture.

\section{ACKNOWLEDGMENT}

The authors wish to thank D. A. Perkins for his assistance in the experiments.

\section{REFERENCES}

[1] J. J. Rocca, J. D. Meyer, and G. J. Collins, "Electron beam pumped CW $\mathrm{Hg}$ ion laser," Appl. Phys. Lett, vol. 40, pp. 300302,1982 .

[2] - "CW laser oscillations in $\mathrm{Cd}$ II in an electron beam created plasma," Phys. Lett., vol. 90A, pp. 358-360, July 1982.

[3] "Electron beam pumped CW Se II laser," Opt. Commun., vol. 42, pp. 125-127, June 1982.

[4] J. D. Meyer, J. J. Rocca, Z. Yu, and G. J. Collins, "CW iodine laser excited by an electron beam," IEEE J. Quantum Electron., vol. QE-18, pp. 326-327, Mar. 1982

[5] J. J. Rocca, J. D. Meyer, and G. J, Collins, " $\mathrm{Zn}$ II As II laser tran- 
sitions excited by an electron beam," IEEE $J$. Quantum Electron., vol. QE-18, pp. 1052-1054, July 1982.

[6] J. J. Rocea, J. D. Meyer, Z. Yu, M. Farrell, and G. J. Collins, "Multi kilowatt electron beams for pumping CW ion lasers," Appl. Phys. Lett., vol. 41, pp. 811-813, Nov. 1982.

[7] J. J. Rocca, J. Meyer, M. R. Farrell and G. J. Collins, "Glow discharge created electron beams: Cathode materials, electron gun designs and applications," unpubiished.

18] Z. Yu, J. J. Rocca, J. Meyer, and G. J. Collins, "Ttansverse electron guns for plasma excitation," J. Appl. Phys, vol. 53, pp.
$4704-4710$, July 1982

[9] K. Rozsa, M. Janossy, J. Bergou, and L. Cllag, "Noble gas mixture CW hollow cathode with internal anode system," Opt. Commun, vol. 23, pp. 15-18, 1977.

[10] T. lijima, "New type hollow cathode discharge tube with continuously variable voltage," Japan. J. Appl. Phys., vol. 20 , pp. L470-L472.

[11] H. Kano, T. Shay, and G. J. Collins, "A second look at the excitation mechanism of the He-Hg' laser," Appl. Phys. Lett., vol. 27, pp. 610-612, Dec. 1975 .

\title{
Compact Sealed TEA $\mathrm{CO}_{2}$ Lasers with Corona-Discharge Preionization
}

\author{
R. MARCHETTI, E. PENCO, AND G. SALVETTI
}

\begin{abstract}
Optimization of the performance of compact sealed TEA $\mathrm{CO}_{2}$ lasers employing a novel version of the coronadischarge preionization scheme is reported. Performance with high oxygen concentrations, long-ife operation with organic additives, limits of arc-free operation with gas mixtures without helium, and in the presence of high $\mathrm{CO}_{2}$ concentrations have been investigated. Our results show that devices whose construction is considerably simplified by employing this preionization technique are capable of producing performance superior to more complex systems.
\end{abstract}

\section{INTRODUCTION AND BACKGROUND}

$\mathrm{T}$ HERE is at present considerable interest in the development of compact pulsed atmospheric-pressure UV preionized $\mathrm{CO}_{2}$ lasers capable of operating at medium/high repetition rates for long periods without the need for gas replenishment. One of the major problems to be faced in order to meet these requirements is the $\mathrm{CO}_{2}$ dissociation in the discharge with concomitant formation of molecular oxygen which gives rise to strongly attaching neutrals and negative ions with subsequent loss of discharge stability [1]-[3]. A solution to this problem has been sought in terms of removal of the molecular oxygen, either by including gaseous additives in the gas mixture [4], [5], or by using hot and cold catalysts [6]-[8], whose utilization is, nevertheless, generally detrimental to simplicity and compactness. Another approach that can be adopted is to use a discharge scheme which minimizes the $\mathrm{CO}_{2}$ dissociation rate in the gas mixture. In fact, this rate is affected by a number of factors, one of the most important being the type of preionization source employed. To this regard, the widely used slidingspark preionization technique [9] has to be avoided, while diffuse-discharge preionizing structures should be preferred since it is well established that they result in a lower $\mathrm{CO}_{2}$ dissociation rate $[2]$.

Manuscript received April 18, 1983.

R. Marchetti and E. Penco are with the Department of Electrooptics, Selenia S.p.A. Via dei Castelli Romani 2, Pomezia, Italy,

G. Salvetti is with ENEA-CRE Casaccia, Laboratorio Sviluppo Processi Fotoassistiti, Via Anguillarese $\mathrm{km} \mathrm{1.300,} \mathrm{Rome} \mathrm{A.D.,} \mathrm{Italy.}$
Among these structures, a most interesting one relies on the generation of preionizing UV photons from a corona discharge over the surface of a dielectric [10], [11]. The corona discharge, generally longitudinally extended for the entire length of the discharge electrodes, produces a very homogeneous UV radiation flux, which is capable of initiating on nanosecond time scales very uniform glow discharges with considerable enhancement of spatial uniformity of the optical gain. This feature obviates the need for using additional pulsing, timing, and switching circuits to allow plasma recombination processes to homogenize the photoelectron distribution prior to the application of the main voltage pulse, as in the case of slidingspark preionized discharges. It is therefore possible to employ very simple electrical configurations where the corona discharge starts during the step up of the voltage applied to the main discharge electrodes and illuminates the gap throughout the whole discharge time, having very little delay and no jitter with respect to the main discharge. This near simultaneous UV preionization scheme makes the laser system fairly insensitive to relatively large concentrations of attaching species (e.g., $\mathrm{O}_{2}$ ), such as those occurring in sealed systems after several million pulses. In addition to this, the corona-type preionization eliminates the problems associated with the erosion of the sliding. spark electrodes, i.e., sputtering of extraneous materials into the laser gas mixture and variation of the electrical characteristics of the preionization discharge with the number of shots.

In this letter, we describe some aspects of the design, operation, and performance of a series of compact sealed TEA CO laser systems which use an improved and simplified version [12] of the preionization technique based on corona-discharges over the surface of a dielectric. With a proper choice of the dielectric material and preionization electrode geometry, efficient UV radiation can be generated from an extremely simple, inexpensive, and rugged photon source. For the optimization of this technique, we have profited from the experience gained in a comprehensive study of the mechanisms involved in photoelectron production by corona discharges, reported in a separate publication [13]. Details of the construction and operat- 